The clinical characteristics were reviewed in 34 patients, age 5-35 years (Wing L. Psychol Med Feb 1981: 11:115-129). Several children walked at a normal age but were slow to talk and became increasingly odd and withdrawn in personality. A genetic factor is suspected and no specific organic pathology has been identified. The syndrome listed in the ICD10 but not in DSM-III-R is sometimes classified as a subgroup of the autistic spectrum with motor clumsiness, higher IQ and higher verbal skills (Tuchman R F. Autism: Delineating the spectrum. Int Pediatr 1991: 6:161-169). Studies involving MRI and possible genetic or environmental factors require further investigation. In the absence of a specific etiology and biological marker, diagnosis is dependent on clinical criteria, a careful history, and full psychiatric and pediatric neurology evaluation.

\title{
ORGANIC BRAIN DYSFUNCTION AND AUTISM
}

A population-based neurobiological study of 35 children with autistic disorder (AD) and 17 with autistic-like conditions (ALC) is reported from the Department of Child and Adolescent Psychiatry, University of Goteborg, Sweden. Major indications of brain damage or dysfunction were found in $90 \%$. Etiological groups included Moebius syndrome (9\%), fragile X syndrome (6\%), chromosomal anomalies (6\%), neurocutaneous disorders (6\%), congenital hydrocephalus (3\%), Rett syndrome (3\%), Laurence-Moon-Biedl syndrome (3\%), severe perinatal distress (9\%), and epilepsy or severe EEG pathology (20\%). Genetic factors were implicated in $9 \%$ with fathers and a brother with Asperger's syndrome. The EEG was abnormal in 50\% and 18 (40\%) had epileptiform discharges, maximal in the temporal lobes. CAT scan abnormalities in $25 \%$ included dilated ventricles, porencephaly, and general atrophy. BAERs were abnormal in 33\%. (Steffenburg S. Neuropsychiatric assessment of children with autism: a population-based study. Dev Med Child Neurol June 1991; $\underline{33}: 495-511)$.

COMMENT. Multiple biological etiologies for autism and autistic-like disorders are suggested by this comprehensive study. Despite the extra effort and patience involved, the pediatric neurologist should not dismiss the child with autistic symptoms to the care of the psychiatrist without first attempting a full neurologic evaluation including EEG and CT or MRI.

\section{COGNITIVE IMPAIRMENT AND CARBAMAZEPINE}

The effects on cognition of carbamazepine, phenytoin and sodium valproate were compared in 64 new cases of childhood epilepsy treated at the Leeds General Infirmary, England. The children age 5-14 years had no neurological deficit and were allocated randomly and assessed prospectively over a 12 month period. They were seizure-free throughout the 12 months. Psychological tests included visual recall, auditory recall (memory), visual scanning (vigilance), Stroop test (concentration), speed of information 
processing, and standard scales of intellectual functioning and reading. Serum levels of carbamazepine (CBZ) showed a significant negative correlation with changes in scores on the sum of five memory tests from before medication to 6 months and a year later. No significant correlations with memory scores were found for children treated with phenytoin (PHY). Only 10 of 126 serum level estimations of CBZ gave a value greater than $8.2 \mathrm{mcg} / \mathrm{L}$. In the sodium valproate (SV) group significant positive correlations were found between serum levels and the sum of five memory tests at 1 month and at 6 months after starting treatment. The memory test clearly showed that there was impaired recent recall on CBZ apparent by 6 months of treatment and even more definite after a year. Reading scores were also lower in the CBZ compared with the PHY group after a year on drug treatment. Doses of CBZ were usually in the lower half of the accepted therapeutic range of 5-15 $\mathrm{mcg} / \mathrm{L}$ (Forsythe I et al. Cognitive impairment in new cases of epilepsy randomly assigned to carbamazepine, phenytoin and sodium valproate. Dev

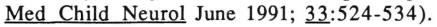

COMMENT. In this study involving children with epilepsy previously untreated, carbamazepine in moderate dosage adversely affected memory but sodium valproate and phenytoin did not. The tendency to favor carbamazepine in preference to phenytoin on the basis of reported cognitive deficits seems questionable and unfounded. A phenytoininduced improvement in auditory memory has been demonstrated in children with EEG dysrhythmias (Millichap J G et al. Auditory perceptual deficit correlated with EEG dysrhythmias. Response to diphenylhydantoin sodium. Neurology 1969; 19:870-872).

\section{METABOLIC AND DEGENERATIVE DISORDERS}

\section{CHILDHOOD DEGENERATIVE DISORDERS AND ADULT DEMENTIA}

An approach to the evaluation of dementia in adults related to pediatric metabolic and degenerative diseases is reviewed from the Department of Neurology, University Medical Center, Maywood, IL. A list of 17 neurodegenerative disorders that ordinarily occur in childhood but may be present in adults includes adrenoleukodystrophy, metachromatic leukodystrophy, Krabbe's disease, Alexander's disease, Lafora's disease, Kufs' disease, mucopolysaccharidosis, Gaucher's type 1, Niemann-Pick disease, and gangliosidosis 1 and 2. Diagnosis is important for genetic counseling and some are treatable. A history of myoclonus suggests Kufs' disease, Lafora's disease, and mitrochondrial disorders. Easy tanning on sun exposure suggests adrenoleukodystrophy. Bone pain occurs with Gaucher's disease. Extrapyramidal symptoms suggest gangliosidosis. Muscle weakness is seen with gangliosidosis and mitochondrial disorders. Depressed deep tendon reflexes may occur with metachromatic leukodystrophy, 